\title{
Gislinde Seybert, Geschichte und Zeitlichkeit. Histoire et Temporalité
}

Annarosa Poli e Stefano Genetti

\section{Q OpenEdition}

1 Journals

\section{Edizione digitale}

URL: http://journals.openedition.org/studifrancesi/9604

DOI: 10.4000/studifrancesi.9604

ISSN: 2421-5856

\section{Editore}

Rosenberg \& Sellier

\section{Edizione cartacea}

Data di pubblicazione: 1 décembre 2007

Paginazione: 680-683

ISSN: 0039-2944

\section{Notizia bibliografica digitale}

Annarosa Poli e Stefano Genetti, «Gislinde Seybert, Geschichte und Zeitlichkeit. Histoire et Temporalité», Studi Francesi [Online], 153 (LI | III) | 2007, online dal 30 novembre 2015, consultato il 14 janvier 2021. URL: http://journals.openedition.org/studifrancesi/9604; DOI: https://doi.org/10.4000/studifrancesi. 9604

Questo documento è stato generato automaticamente il 13 janvier 2021.

\section{(c) (1)}

Studi Francesi è distribuita con Licenza Creative Commons Attribuzione - Non commerciale - Non opere derivate 4.0 Internazionale. 


\title{
Gislinde Seybert, Geschichte und Zeitlichkeit. Histoire et Temporalité
}

\author{
Annarosa Poli e Stefano Genetti
}

\section{NOTIZIA}

GISLINDE SEYBERT, Geschichte und Zeitlichkeit. Histoire et Temporalité, Internationales pluridisziplinäres Kolloquium. George Sand zum Bicentenaire 2004, Bielefeld, Aisthesis Verlag, 2007, pp. 219.

1 Curato da Gislinde SEYBERT, il volume raccoglie i dodici contributi presentati al convegno tenutosi presso l'Università di Hannover in occasione del bicentenario della nascita di George Sand. Come sottolinea la curatrice nell'introduzione (Einleitung, pp. 9-13), essi vertono ora su alcune costanti di tutta l'opera ora su singoli testi appartenenti a diversi periodi. Vari strumenti interpretativi - filosofia, psicocritica, pensiero femminile e femminista, intertestualità ed estetica della ricezione convergono in una riflessione sulla rappresentazione della storia, sulla configurazione e sulla prefigurazione della temporalità e dell'atemporalità.

2 Bernard Hamon, in Anticatholicisme et anticléricalisme dans le roman sandien (pp. 15-33), esamina due aspetti della opposizione di G. Sand a certi principi della religione cattolica in cui era stata cresciuta: l'anticattolicesimo e l'anticlericalismo. Il primo si sviluppa notevolmente in lei a partire dal 1840. Insieme a Pierre Leroux crede nel progresso dell'umanità sulla conoscenza del creatore nel segno dell'uguaglianza. Le generazioni sono volte verso sempre nuove rivelazioni, i vangeli quindi non rappresentano che un momento della rivelazione in continuo sviluppo e anche Gesù Cristo ha diffuso una dottrina incompleta in attesa d'un nuovo messia pur essendo considerato un «philosophe sublime» e un "prophète de l'idéal». Questa negazione della divinità del Cristo appare evidente nella seconda Lélia e in Spiridion. Anche il clero viene condannato perché sarebbe stato opportuno tornare agli ordini monastici del medioevo che hanno contribuito alla trasmissione del sapere senza ambizioni terrestri e lotte 
interne. L'adesione di G. Sand al vangelo di San Giovanni si spiega soprattutto per il suo valore epico e sociale che ritrova nelle dottrine di Pierre Leroux. Se il cattolicesimo va verso la rovina totale, vittima dei suoi errori, per la scrittrice ora occorre aspettare a prospettare un nuovo credo al fine di evitare un individualismo senza freni che potrebbe solo distruggere il sogno di libertà, uguaglianza e fraternità. Il soggiorno a Roma di Sand nel 1855 le permette di constatare le penose condizioni di vita della città governata dal potere clericale e di dimostrare il suo anticlericalismo nel romanzo $L a$ Daniella, nel 1847 e, nel 1863, in Mademoiselle La Quintinie. Alla prima del Marquis de Villemer nel 1864, gli studenti del Quartiere latino manifestarono contro i clericali per sostenere la scrittrice che si era opposta alla politica di Napoleone III, troppo compiacente verso la Chiesa cattolica.

3 Secondo Michel ARDUimi (Le secret de Sand dans "Le Chateau de Pictordu", pp. 35-48), in questo racconto, tratto dai Contes d'une grand-mère, il tema dell'arte è visto in una prospettiva storica. I personaggi che vivono alla fine del Settecento risentono sia sul piano dell'arte che su quello psicologico della mutazione del gusto tra il rococò e lo stile neoclassico in un ritorno all'antichità che coincide con la ricerca della coscienza poetica. L'indagine storica è associata al conflitto di Diana con il padre che le ha imposto una matrigna odiosa e non è per lei quel rifugio che avrebbe voluto trovare. Il suo desiderio di dipingere copiando figure da altri quadri per ritrovare i tratti della madre morta, rappresenta la finalità catartica dell'arte nell'indagine sul «segreto della vita». L'autore mette in evidenza come Sand abbia delle aspirazioni esoteriche legate alle sue scelte spirituali che sono incompatibili con la presa di coscienza di un Baudelaire o di un Rimbaud. E pertanto la scrittrice si rivela moderna nella svalutazione dell'idea del «Numero» quando Blanche si mostra pronta a vendere il castello di Pictordu e lei non le offre denaro, ma monete rarissime che superano il valore espresso in denaro.

4 Nell'analizzare il medesimo testo della sessantottenne George Sand, Thomas Amos ( $L a$ Poesie voilée. Meta-Fiktionalität des Märchens in George Sands "Le Chateau de Pictordu", pp. 49-82) insiste sulla dimensione autoriflessiva, metanarrativa e metafinzionale, oltre che autobiografica, sulla dimensione intermediale oltre che intertestuale di un racconto che, all'insegna delle corrispondenze tra pittura e letteratura, associa merveilleux e satira sociale, modalità fantastica e racconto di formazione. Analizzando i contorni ideologici dei personaggi, le valenze autoreferenziali di episodi e passi descrittivi, l'autore ricostruisce le tappe dell'iniziazione all'arte della piccola Diane. Mostra come il racconto suggerisca un'innovativa poetica della fiaba, tesa a superare ogni distinzione tra letteratura per l'infanzia e per adulti, propugnando un'estetica impegnata, che sintetizza istanze romantiche e realiste in contrasto con la concezione superficiale dell'arte incarnata dal padre della protagonista, peintre de salon.

5 Nel roman champêtre Jeanne - argomenta Annette RUNTE ("Singende Steine". Zur Verschränkung von Mythos und Geschichte in George Sand's Landroman "Jeanne”, pp. 83-109) - Sand rivisita il motivo del sacrificio simbolico della vergine all'insegna di un realismo poetico che contesta la distinzione tra idealismo femminile e pragmatismo maschile. Riarticolando storicizzazione del mito e mitizzazione della storia, la légende rustique contiene una riflessione sulla condizione della donna in seno alla società patriarcale $\mathrm{e}$ sulle origini della consapevolezza della differenza di genere.

6 Gérard CHALAYE (Intention de l'Hérésie ou la double temporalité primitive dans "Consuelo" et "Jean Ziska", pp. 110-137) si chiede fino a che punto l'eresia sandiana è la riscoperta di 
un fatto storico o piuttosto l'invenzione di un mito. Cita le opere che hanno maggiormente influenzato Sand nelle sue convinzioni sul concetto di eresia a partire dalla Encyclopédie nouvelle di Pierre Leroux e, per quanto riguarda l'eresia hussita, in particolare in Consuelo e in Jean Ziska. In Spiridion dominano invece le teorie del «joachinisme» e del «johannisme» così diffuse in quel tempo anche nelle logge massoniche. La storicizzazione della religione, sostenuta da Gioacchino de Fiore, aveva sedotto i romantici, persuasi della evoluzione progressiva dei principi religiosi che, secondo Sand, avrebbero dovuto confluire in un credo umanitario espresso da «L'Évangile Éternel». È l'origine di quelle che saranno le opinioni di Vico, Hegel, Comte, Marx. Sand proclama più chiaramente di Joachim la morte della religione cristiana ed è così evidente l'importanza che Jean Huss assume come figura emblematica agli occhi della scrittrice. Le figure mitiche di Jean Ziska e Procope le Grand, ambedue martiri delle libertà, sono all'origine dell'ultima grande eresia che è quella della Rivoluzione francese. Come Michelet, Sand pensa che Satana sia l'agente essenziale della rivolta sociale e non più un principio negativo. Così si attua una «riparazione» metafisica $\mathrm{e}$ storica. Chalaye conclude questa sua minuziosa e acuta ricerca affermando che G. Sand, in quanto scrittrice, diventa la profetessa della «religione delle religioni», «la religion éternelle», capace di unificare tutte le altre e di dare loro un significato. Si può dunque pensare a una «invention de l'hérésie» in quanto mito produttivo di una nuova storia.

7 Per Béatrice DIDIER ("Nanon", roman de l'évolution, pp. 131-138), diversamente dagli altri romanzi in cui la «voce narrante» è maschile, in Nanon il racconto è imperniato su una donna dalla notevole personalità che evoca a forti tinte la Rivoluzione francese. Nanon è una contadina che non per ambizione, ma solo per amore riesce a sposare un giovane aristocratico. Mai rinnegherà le sue origini contadine, sempre vestita con gli abiti tradizionali del Berry. Desidera migliorare la sua condizione sociale, la sua cultura, senza volersi affermare come una "pasionaria della Rivoluzione» come aveva cercato di fare la stessa Sand nel 1848, pur restandone profondamente delusa. Nanon rappresenta la Francia contadina, più lenta di quella delle grandi città. A causa della rivoluzione ci si rende conto che esiste una Bastiglia, che c'è in corso una guerra e che si possono comperare i terreni e vendere i Beni nazionali senza violenza, anche se si avverte una evoluzione. B. Didier conclude con la sua consueta penetrante intuizione che il merito di Sand è quello di dare un apporto personale a quella lunga riflessione sul 1789 in atto per tutto l'Ottocento.

8 A partire dalla duplice nozione di désir du texte - il desiderio cui il testo risponde e quello che suscita - Gislinde SEYBER (Textbegehren im Werk von George Sand, pp. 139-147) interroga una scelta di testi sandiani - alcuni capitoli di Lélia, le Lettres d'un voyageur e la prosa poetica di À l'Ange sans nom - sulla scia del metodo di Charles Mauron. Individua così, intorno all'immagine ossessiva, funerea e monumentale, della statua di marmo bianco, un reticolo metaforico associato a rimandi biblici, un mito personale che coniuga amore e morte, colpa ed espiazione.

9 Lélia è un personaggio complesso: donna bella e fatale, ma incapace di amare fisicamente, è soprattutto una intellettuale. Gérard GOEBEL ("Lélia" et "Poliphile", pp. 149-153) si chiede se sia piuttosto un Laelius, amico di Scipione l'Africano al femminile che viene descritto con delle caratteristiche comuni a quelle di Lélia. Ci potrebbe essere anche una correlazione con il personaggio di Polias nel Sogno di Polifilo che in realtà si chiamava Lucrezia, e apparteneva alla nobile famiglia Laelia dell'antichità. Un eco de L'Embarquement pour Cythère si ritrova nella descrizione del sogno ricorrente di Sand 
che si vedeva seduta su una riva deserta mentre degli amici venivano verso di lei su una barca cantando delle «arie deliziose». Ma, mentre il Sogno di Polifilo, unisce l'amore spirituale e quello carnale, in Lélia l'amore spirituale vince su quello fisico. Ritroveremo anche il nome di Pulchérie, sorella di Lélia nel romanzo sandiano nel Sogno di Polifilo, come antico soprannome di Venere. G. Goebel conclude affermando che Mallarmé parla di «un sépulcre qui rit de porter ce nom Pulchérie» poiché nel Sogno di Polifilo è Adone che muore e non Pulchérie. Proprio questa allusione ha ispirato l'autore per il paragone tra Lélia e il celebre Sogno. Sarebbe stato opportuno aggiungere nelle note la referenza all'articolo di Maria Luisa Belleli, Armonia di struttura e coerenza psicologica in "Sylvie" di G. de Nerval (Torino, Giappichelli Editore, 1970, p. 19), dove si parla della Hypnerotomachia Poliphili di Francesco Colonna e del libro di Franco Bacchelli, Un Enigma bolognese. Le molte vite di Laelia Crispis (Bologna, Costa Editore, 2000).

Olga KAFANOVA studia L'Autobiographie de George Sand comme texte dialogique dans la réception russe du XIX siècle, pp. 154-170). Histoire de ma vie (1847-1855) non è una vera $\mathrm{e}$ propria storia della sua vita: lo riconoscono i vari biografi di Sand quando si chiedono le ragioni delle omissioni di avvenimenti delle date inesatte e delle successioni dei fatti imprecisi. In realtà si tratta di un'opera che descrive in modo veritiero il passaggio dal Settecento all'Ottocento poiché è più dedicata al passato che al presente. Il grande interesse di queste Memorie è che il lettore vede svilupparsi un pensiero che risale al tempo di Voltaire per giungere al sogno di un'umanità che ha trovato la sua vera strada nella realizzazione ideale della fraternità e della giustizia. Che importano gli amori della romanziera quando attraverso il dialogo con i vari amici e il dialogo mentale interiore riesce a far comprendere la transizione delle idee tra un secolo e un altro? La severità dell'aristocratica nonna paterna verso sua madre, figlia del popolo, è descritta in modo crudo, ma sembra far comprendere le mentalità del tempo come conferma anche «la réception russe» di queste Memorie. L'autore sostiene che nelle varie discussioni proprio questa «réception» è servita ad arricchire l'opera nel dialogo tra testo e lettore, tra la cultura russa e quella francese, anche se Histoire de ma vie sta per essere tradotta in russo solo ai nostri giorni.

11 Yvette FORMERY (George Sand et le temps qui passe, pp. 171-180), descrive una personalità secondo diversi approcci: psicologico, sociologico ecc. Sand si interessava alla fisionomica attraverso l'opera di Lavater. Secondo l'autore, la romanziera, secondo la terminologia caratterologica, potrebbe inserirsi nella famiglia dei «collerici». Anche se Histoire de ma vie dà una grande importanza al passato della sua famiglia, la sua esistenza personale concede poco spazio ai rimpianti e alla nostalgia perché è tutta occupata dal presente. Anche la sua opera è segnata, attraverso una grande immaginazione, dall'ardore delle passioni e delle azioni. Attraverso i giudizi di amici e di scrittori contemporanei l'attività di Sand si manifesta attraverso una impetuosa mobilità derivata dalla sua attività fisica e da quella della sua immaginazione. In lei domina l'emotività e un'attività incessante. Il «collerico» non si preoccupa di avere una vita coerente, ma gli basta avere una vita esuberante. Non sacrifica un amore all'altro, ma aggiunge il secondo al primo. Questa incostanza della scrittrice ha urtato negativamente chi ha voluto giudicarla, non a causa del moralismo ma di una divergenza profonda nel concetto di sentimento. Quando la romanziera cambia amori e crede di essere guarita dei suoi errori è proprio il momento in cui è spinta a ricominciare. Anche nella sua opera si mostra impulsiva, violenta, impaziente e prende diverse direzioni: romanzo, teatro, storia. Detesta i rimpianti e la malinconia ed è dominata da una volontà di superare le difficoltà, di non dipendere né dalla tradizione 
né dall'opinione. Nella prefazione di Consuelo dichiara di procedere nel suo argomento giorno per giorno. La concezione della storia per Sand è il riflesso della sua emotività dominante come risulta da questa sua affermazione: «L'histoire se sert [...] de tout, d'une note de marchand, d'un livre de cuisine, d'un mémoire de blanchisseuse». Domina in lei una tendenza all'aggressività e al dominio su quelli che le erano vicini. Manifestò una vivace aspirazione a realizzare e a soddisfare tutti gli aspetti del suo destino di donna fino alla fine della sua vita. Y. Formery fornisce qui un'interpretazione personale del carattere della romanziera francese, ma che potrebbe anche essere discussa.

Gisela DISCHNER (Der Ausstieg aus der Zeitlichkeit: Mystische Spuren im Werk der George Sand, pp. 181-190), evocando antecedenti e mobilitando raffronti che vanno da Eraclito a sant'Agostino e da Hölderlin a Nietzsche e a Rilke, rinviene nell'opera di Sand, le tracce mistiche collegabili al kairos, all'istante privilegiato sottratto alla cronologia, tempo senza tempo dove passato, presente e futuro si fondono così come interiorità ed esteriorità, soggetto e oggetto. Tra estasi e allucinazione si delinea un pathos avveniristico, l'aspirazione a un rinnovamento dell'umanità le cui variegate implicazioni utopistiche, religiose, sociali e politiche richiamano il programma idealistico del primo romanticismo tedesco.

Laura cоLомво (La Liberté guidant les femmes: George Sand, Marie d'Agoult et l'Histoire au féminin au XIX ${ }^{e}$ siècle, pp. 191-219) evoca il celebre ritratto della libertà che guida il popolo nel quadro di Delacroix che raffigura una donna dal fisico possente e questa rappresentazione vigorosa simbolizza la visibilità che chiedono le donne dell'Ottocento nella loro scrittura sia che tratti di politica che di storia. Le loro ambizioni vanno al di là delle storie private degli amori rappresentati nei loro romanzi poiché esse vogliono conquistare il loro posto utilizzando vari generi letterari. Spesso si richiamano alle Memorie delle donne celebri del passato e si inseriscono fra i «pensatori» della storia. Dopo il 1830 le prime giornaliste giudicano l'attualità maschile senza rinunciare a un ruolo didattico di fronte alle donne. Amélie Bosquet, prima di Sand, si volge verso il folklore, Hortense Allart si occupa di critica storica e dedica uno studio monumentale alla storia europea. Sand, pur rifiutando di essere la rappresentante politica delle donne, si getta nella mischia redigendo i Bulletins de la République con lo scopo di fare opera di propaganda per la repubblica così come nei suoi articoli pubblicati sulla Cause $d u$ peuple. Donne che dominano i salotti culturali, donne che redigono come Marie d'Agoult (Daniel Stern) una Histoire de la Révolution de 1848. Questa aristocratica che rivendica i diritti del nuovo proletariato industriale resta tuttavia sempre legata alla classe dalla quale deriva. Vede negli studi sulle scuole socialiste una prevalenza di utopie e cita Proudhon in opere come Essai sur la liberté e Pensées, réfléxions et maximes. L'autore analizza le opere storiche di Marie d'Agoult anche dal punto di vista stilistico. La ricostruzione epica di Daniel Stern viene confrontata con quella di Hugo nel comune desiderio di una conciliazione ad ogni costo. Nella sua Histoire M. d'Agoult dedica alla rivoluzione europea un lungo capitolo, come dedica spazio alle rivendicazioni delle femministe pur distinguendosi da esse: per queste scrittrici non era ancora giunto il momento della rivoluzione generalizzata, ma il germe della solidarietà femminile era stato gettato. Concludendo questo interessante studio sulle donne e la libertà nel secondo Ottocento l'autore sostiene che esse recano una visione nuova della storia attraverso la resurrezione di una genealogia femminile da attuarsi in un'opera collettiva e corale, alla ricerca della solidarietà e della perfettibilità, con un'operazione che tiene conto delle micro-storie. È il solo modo per le donne del momento di uscire 
dal ruolo di icone escatologiche rappresentato dagli artisti e dai poeti per offrire il loro contributo al miglioramento dell'opera dell'umanità. 